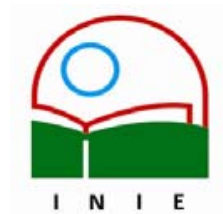

Actualidades Investigativas en Educación

Revista Electrónica publicada por el

Instituto de Investigación en Educación

Universidad de Costa Rica

ISSN 1409-4703

http://revista.inie.ucr.ac.cr

COSTA RICA

\title{
EL NÚCLEO PEDAGÓGICO: UNA EXPERIENCIA INTEGRADORA DE INVESTIGACIÓN, DOCENCIA Y ACCIÓN SOCIAL EN LA ESCUELA DE TRABAJO SOCIAL
}

THE PEDAGOGIC NUCLEUS: AN INTEGRATIVE EXPERIENCE OF RESEARCH, TEACHING AND SOCIAL ACTION IN THE SCHOOL OF SOCIAL WORK OF THE COSTA RICAN

Volumen 7, Número 1

Enero-Abril 2007

pp. $1-20$

Este número se publicó el 30 de abril 2007

Ivette Campos Moreira

La revista está indexada en los directorios:

LATINDEX, REDALYC, IRESIE, CLASE, DIALNET, DOAJ, E-REVIST@S,

La revista está incluida en los sitios:

REDIE, RINACE, OEI, MAESTROTECA, HUASCARAN

Los contenidos de este artículo están baj o una licencia Creative Commons 


\title{
EL NÚCLEO PEDAGÓGICO: UNA EXPERIENCIA INTEGRADORA DE INVESTIGACIÓN, DOCENCIA Y ACCIÓN SOCIAL EN LA ESCUELA DE TRABAJO SOCIAL \\ THE PEDAGOGIC NUCLEUS: AN INTEGRATIVE EXPERIENCE OF RESEARCH, TEACHING AND SOCIAL ACTION IN THE SCHOOL OF SOCIAL WORK OF THE COSTA RICAN
}

\author{
Ivette Campos Moreira ${ }^{1}$
}

\begin{abstract}
Resumen: Este artículo presenta las experiencias que la Escuela de Trabajo Social ha desarrollado en relación con la puesta en práctica de procesos pedagógicos de integración, docencia, investigación y acción social (NIDIAS). Estos son concebidos como una estrategia institucional para potencializar una docencia que se enriquezca con la investigación y viceversa. Pero al mismo tiempo desencadene procesos de servicios que contribuyan a generar cambios en los espacios de la vida social con los participantes (docentes, estudiantes, profesionales y las personas que acuden a los servicios estatales y no estatales) y en sus propios espacios comunales y familiares.
\end{abstract}

Se culmina con la presentación de algunas lecciones aprendidas en relación con los aspectos teóricometodológicos constituidos como componentes básicos para el enriquecimiento de la puesta en práctica de los núcleos integradores de docencia, investigación y acción social (NIDIAS).

Palabras clave: PEDAGOGÍA, DOCENCIA, INVESTIGACIÓN, ACCIÓN SOCIAL, FORMACIÓN ACADÉMICA

\begin{abstract}
This article present the experiences that the school of social work of The Costa Rican University has developed in relation to the execution of pedagogic processes of integration of the teaching research and social action (NIDIAS). These are conceived as an institutional strategy to foment a teaching that gets rich with the research and vice versa. But at the same time unchain processes of services that contribute to generate chances in the spaces of the social life with people that participate such as (educational, students professionals and people that go to the state and not state services) and in their own communal and family spaces.
\end{abstract}

You conclude with the presentation of some lessons learned in connection with the methodological theoretical aspects that are constituted in central components for the enrichment of the setting in practice of the integrative nuclei of teaching, research and social action (NIDIAS)

Keywords: PEDAGOGY, TEACHING, RESEARCH, SOCIAL ACTION ACADEMIC FORMATION

\footnotetext{
${ }^{1}$ Master en Administración Publica, Licenciada en Trabajo Social ambos títulos de la Universidad de Costa Rica. Profesora e Investigadora de la Escuela de Trabajo Social y de la Maestría en Trabajo Social, Universidad de Costa Rica.
}

Correo electrónico: ivettecamposm@yahoo.com

Artículo recibido: 5 de febrero, 2007

Aprobado: 25 de abril, 2007 


\section{Introducción}

Dos argumentos importantes se constituyen en razones institucionales y profesionales para la realización de esta propuesta pedagógica. Uno se relaciona con la necesidad de articular los esfuerzos académicos que en las décadas pasadas y en el momento actual ha realizado la Escuela de Trabajo Social. Otro está vinculado con la necesidad de generar procesos potencializadores de docencia-investigación y la acción social. Esta estrategia es denominada en el Plan Estratégico 2006-2010: Núcleos integradores de docencia investigación (NIDIAS).

Los núcleos son un esfuerzo colectivo de estudiantes, profesores y profesionales que, mediante prácticas académicas, trabajos finales de graduación, investigaciones y talleres de actualización desde el grado y el posgrado, pueden contribuir a generar conocimiento en distintas áreas. Se busca con esta estrategia subsanar la práctica predominante de trabajos desarticulados de un proyecto profesional de mayor alcance. Sin embargo, tales inquietudes fueron planteadas en otros momentos históricos del desarrollo de la formación académica, lo que desembocó en experiencias de gran valor para las iniciativas actuales. Es importante explicitar la experiencia académica de la Escuela de Trabajo Social, en relación con esta estrategia pedagógica. Este artículo presenta tres experiencias de integración de la docencia y la investigación social que han sobresalido en el último quinquenio. Se culmina con algunas precisiones conceptuales y metodológicas sobre los NIDIAS como una contribución para su puesta en práctica en el próximo quinquenio.

\section{Antecedentes en relación con la integración de la docencia investigación y acción social en los últimos cinco años.}

La Escuela de Trabajo Social, a lo largo de su desarrollo histórico, ha buscado incesantemente fórmulas operativas para alcanzar la integración de las funciones citadas. En el año 1976, se experimenta una fórmula de impacto mediante un proyecto de investigación titulado: ¿Qué piensan, qué hacen y qué sienten los grupos sociales populares en Costa Rica? Este contribuyó a la docencia mediante un conjunto de herramientas teóricometodológicas para el acercamiento a estos grupos, considerados en este momento histórico como el objeto-sujeto de la intervención del Trabajo Social. 
Sin embargo es, en el último quinquenio que se llevan a cabo tres prácticas relevantes que podrían coadyuvar y sentar las bases para las experiencias de los NIDIAS que se plantean como centrales en el plan estratégico del 2006-2010. Estas son:

1. La integración de la investigación, docencia y acción social en torno a la democratización de la vida cotidiana, como alternativa de acción para la atención de la violencia social en San Carlos (Campos y Méndez, 2003).

2. La gestión local en relación con el riesgo de desastres: una experiencia de articulación de la investigación a la acción social (Cerdas, 2005).

3. Articulación de la producción de conocimiento desde los Trabajos Finales de Graduación (TFG) a las áreas críticas como puntos de partida para la investigación docencia y acción social (Campos, Casas, Gutiérrez y Smith, 2003).

\section{La integración de la investigación, docencia y acción social en torno a la democratización de la vida cotidiana, como alternativa de acción para la atención de la violencia social en San Carlos.}

Este proceso se inicia en 1999 y culmina en 2003. Los cursos de taller son las experiencias de aprendizaje que sintetizan procesos de transmisión, producción de conocimiento y acción social. La articulación de este conjunto de tareas académicas se formaliza en un proyecto de investigación denominado "La integración de la investigación docencia y acción sociales en torno a la democratización de la vida cotidiana, como alternativa de acción para la atención de la violencia social". En él se condensan las directrices institucionales y las de la Red Interinstitucional contra la violencia en San Carlos. La reconstrucción de los procesos de investigación y de acción social con las trabajadoras sociales, el aprendizaje con las estudiantes, las supervisiones, la revisión y análisis teórico, se constituyeron en fuentes valiosas para destacar la labor de las profesionales y el aporte de la Escuela de Trabajo Social. La atención profesional, la práctica académica y la investigación representan la dimensión concreta que sirve de eje para la comprensión y la intervención en torno a la violencia que viven las mujeres, niñas, niños, y las familias en las distintas comunidades de San Carlos. 


\subsection{El origen y desarrollo de la experiencia de integración: desde la docencia hacia la investigación y la acción social. Profesionales, profesoras y miembras tomadoras de decisiones acuerdan iniciar la experiencia.}

En el año 1999, se gesta esta alternativa de integración originada en la docencia; se formaliza por medio de la investigación y se culmina en forma alternativa o sucesiva con la acción social. Esta viene a dar respuesta a dos exigencias: una interna y otra externa.

La primera obedece, por una parte, a la necesidad de la Escuela de Trabajo Social de ejecutar proyectos de práctica académicas que integren los diferentes niveles del Plan de Estudio y con ello el desarrollo de prácticas generadoras de un mayor impacto social. Esto permite de alguna manera visualizar la transformación de los estudiantes, los profesores, los profesionales de Trabajo Social y las personas de las comunidades, en torno al estudio y la acción sobre problemas de gran complejidad, como la violencia social, la pobreza, entre otros. Y la externa, de la Red regional contra la violencia ${ }^{2}$ de ampliar su radio de acción a acciones preventivas y promocionales en relación con los problemas citados-constituyen el inicio de un proceso de encuentros para aunar esfuerzos académicos y de acción social.

\subsection{La explicitación de necesidades sociales y académicas: el camino organizativo para su desarrollo.}

La formación académica de Trabajo Social debe asumir la intervención en problemáticas de alta complejidad, tomando en cuenta procesos de conocimiento y de intervención que conciban las interacciones de los fenómenos. La atención debe interpretarse en ese tejido social donde las instituciones actúan a partir de la construcción de vínculos que aseguren la captación de las aristas visibles e invisibles de los problemas sociales. En este sentido, las necesidades que plantean las instituciones participantes en la red contra la violencia, ofrecen el escenario idóneo para el desafío que debe imponerse el Trabajo Social del inicio de siglo. La riqueza de esta experiencia nos permite conocer y escuchar la voz de las mujeres, de los niños y niñas, de los jóvenes, de las familias, de los profesionales, de los líderes, de los pobladores de las comunidades, en forma directa al referirse a sus problemas, sus situaciones de vulnerabilidad, sin la necesidad de encajarlos en categorías a priori. Esta condición destacada la señala Matus (1995) como un desafío, al aunar dos objetivos: lo

\footnotetext{
${ }^{2}$ Esta Red interinstitucional, opera en San Carlos. Su área de atracción es la región Norte del país. Volumen 7, Número 1, Año 2007, ISSN 1409-4703
} 
profesional y lo político, en el sentido de asegurar un control procedural en la toma de decisiones, y escuchar a todos los afectados. Al respecto, los encuentros entre las docentes y las profesionales de la Red confluyen en definir lo siguiente como el escenario común de conocimiento e intervención:

\section{En Docencia}

- Procesos socioeducativos con familias o mujeres que hayan vivido o vivan situaciones de violencia familiar. En estos, las interacciones de los participantes son posibilitadas por la discusión de temas en torno a la violencia y las soluciones desde la perspectiva de estos. Los estudiantes, los profesores y los profesionales de la Oficina de la Mujer, del Patronato Nacional de la Infancia y del Sistema Local de Rehabilitación se constituyen en facilitadores de procesos de interaprendizaje, para la toma de conciencia en torno a las formas de violencia que se originan y se desarrollan en la región y a las potencialidades internas y externas para la transformación de tales formas de convivencia.

- Procesos socioeducativos en espacios locales con los líderes, organizaciones comunales, personas y familias que comparten intereses en comunidades ${ }^{3}$. La priorización de los problemas desde la perspectiva de los pobladores, la búsqueda de soluciones, la asunción de compromisos colectivos con las instituciones que les facilitan servicios para mejorar su calidad de vida, se constituyeron en logros en términos de poseer un mayor control sobre decisiones y manejo de información para atender sus principales necesidades.

- Procesos de interaprendizajes entre profesoras y profesionales de la Red Interinstitucional contra la violencia, en el sentido de crear espacios de reflexión sobre el trabajo en red, la sexualidad, la comunicación y la violencia en la familia y su relación con los derechos humanos.

\section{En Investigación}

- Procesos de investigación relacionados con el conocimiento de las formas perceptibles e imperceptibles de la violencia desde los actores comunales e institucionales.

\footnotetext{
${ }^{3}$ Las comunidades seleccionadas fueron Concepción, Barrio La Cruz, San Pablo de San Carlos. Región Norte. Costa Rica 
- Procesos de investigación para el examen riguroso de las formas de intervención del Trabajo Social en relación con la violencia.

- Reconstrucción de la ruta de servicios institucionales que siguen las personas víctimas de la violencia en San Carlos.

- Construcción de indicadores de violencia para la creación de sistemas de información que se constituyan en materia prima para la toma de decisiones en beneficio de las personas víctimas de situaciones de violencia.

\section{En Acción social}

- Un Trabajo Comunal Universitario titulado Defendiendo los derechos y buscando oportunidades para las mujeres en situación de violencia doméstica.

- Cursos de extensión docente sobre la sistematización de la experiencia del trabajo en red contra la violencia doméstica en San Carlos; Taller sobre La comunicación interpersonal; Taller sobre Familia, Derechos Humanos y violencia; Taller sobre las artes creativas al servicio de las niñas y los niños que han sufrido abuso en sus diferentes modalidades y/o violencia familiar; Taller del Manejo de la Sexualidad en la familia para padres y madres de familia.

- Participación en actividades comunales y universitarias que permitieron la difusión de los resultados del proyecto en la Feria de la Salud en San Carlos y la Expo 2001 en la Universidad de Costa Rica.

El diagrama siguiente destaca los servicios institucionales en red, en los que se generaron procesos de investigación docencia y acción social, como actividades centrales de esta experiencia de integración. 


\section{Red interorganizacional de servicios de las instituciones participantes}

San Carlos 2000-2002
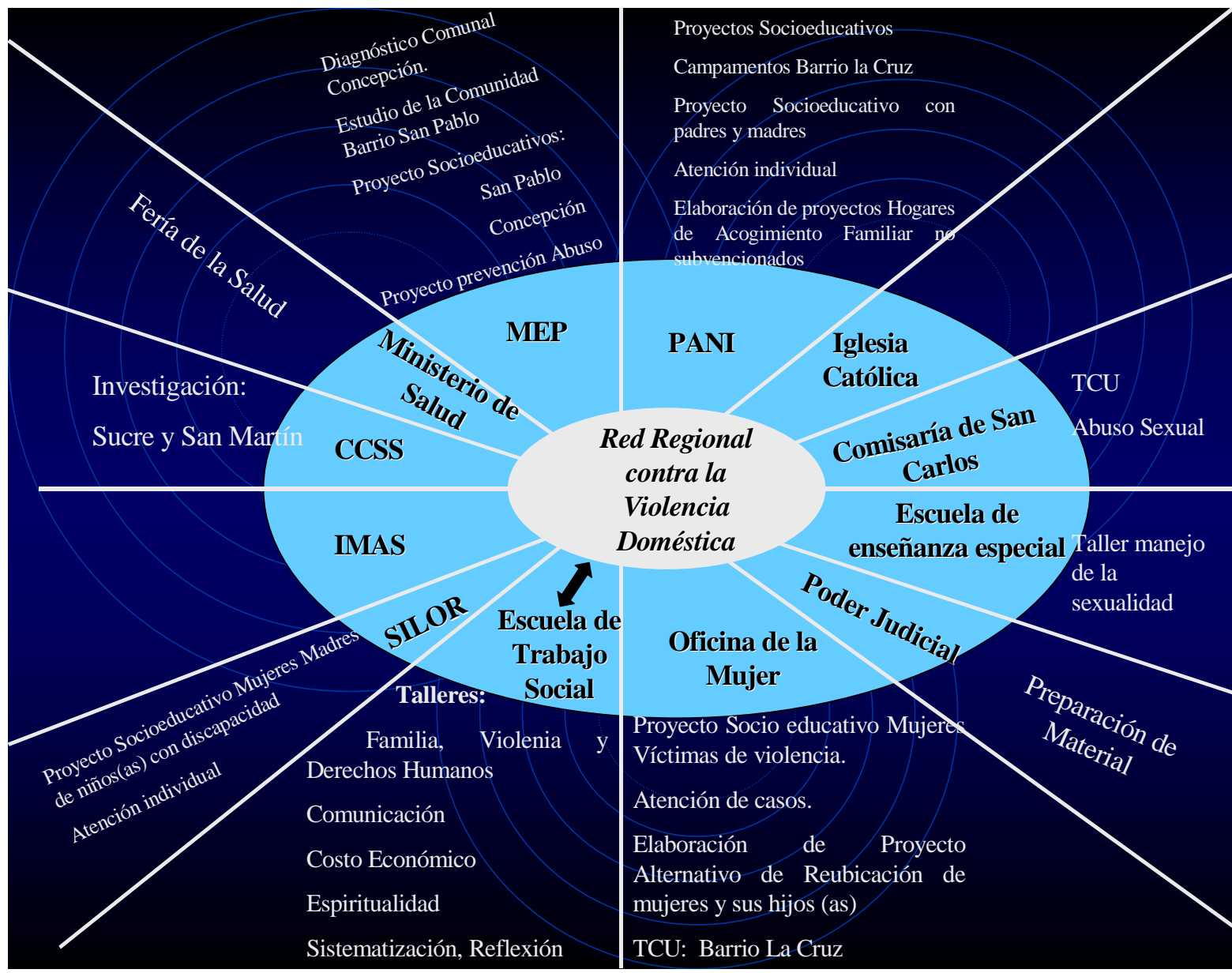

2. La gestión local en relación con el riesgo de desastres: una experiencia de articulación de la investigación a la acción social, con estudiantes de distintas disciplinas, profesoras, organizaciones locales, miembros de la comunidad de Cachí y profesionales de instituciones

La Escuela de Trabajo Social de la Universidad de Costa Rica asume el reto de articular una serie de esfuerzos institucionales y locales para llevar a la práctica un enfoque alternativo que aborde los desastres y genere desde la gestión local procesos participativos de reducción del riesgo.

Dicha experiencia se inició en el año 2004 bajo la modalidad de Acción social con un curso de de Extensión Docente denominado: "Capacitémonos para reducir el riesgo ante los 
desastres en Cachí", con participación de profesores y miembros de dicha comunidad. Esta experiencia se deriva del Trabajo Final de Graduación (TFG): "Si no unimos lo que tenemos nadie va a hacerlo...Conformación de una organización para reducir el riesgo ante los desastres en Cachí, Paraíso, Cartago" (Cerdas, 2004, p. 2).

Lo esencial en esta actividad es la gestión de una alternativa mediante la cual los actores sociales generan una comprensión de la realidad de los desastres como un proceso social e histórico cuyas prácticas cotidianas constituyen la singularidad del proceso total. Esta singularidad permite la captación de los escenarios de riesgo, para asumir una manera alternativa de abordar esa situación, mediada por la conformación de una red local, así como por la gestión de acciones en la reducción de los riesgos.

Lo anterior plantea la necesidad de la participación activa de los actores locales, profesores y estudiantes, en el fortalecimiento de la capacidad organizativa y operativa desde la comunidad de Cachí. El punto de partida es la situación de riesgo que se vive en la zona. Se reconoce la poca respuesta que las autoridades institucionales competentes han brindado a esa situación, lo que también contribuye al análisis y reflexión para la articulación con las organizaciones gubernamentales como actores participantes en la gestión de la atención al riesgo de los desastres en la comunidad de Cachí.

Sobre la base de dicha experiencia, se deriva un proyecto de Trabajo Comunal Universitario a partir del año 2005. La reducción del riesgo ante los desastres en el distrito de Cachí se constituyó en lo esencial para la construcción de alternativas locales que contaron con la participación de:

- 10 actores comunales: representantes del Comité de Emergencias, Asociación de Desarrollo Integral de Cachí, Junta de Educación del Colegio, Asociación de Vivienda, ASOPAC, Asociación de Desarrollo San Miguel, Asociación de Desarrollo San Jerónimo, Comité de Vecinos de Calle Volio y Grupos de la Iglesia Católica. Estos forman parte de la Red Local para la Reducción de Riesgos en Cachí, organización que se encuentra dirigida por una comisión coordinadora; cuenta con unidades operativas como la comisión ambiental, la de planificación urbana, la de activación juvenil y divulgación.

- Un total de 18 estudiantes de las carreras de Geología, Geografía, Ingeniería Civil, Ingeniería Agrícola, Psicología, Comunicación Colectiva, Educación, Derecho y Artes 
Dramáticas, trabajan en conjunto con los actores comunales, a partir de su integración en las comisiones de la Red Local y en equipos multidisciplinarios, en la problematización del riesgo del distrito y en la construcción de alternativas viables para el estudio y participación local en la reducción del riesgo.

Los principales resultados se relacionaron con:

- La participación y continuidad de los actores comunales en procesos formativos para generar conocimiento desde una visión alternativa de los desastres y proponer alternativas para abordar la reducción del riesgo. El interés y compromiso para llevarlas a cabo se constituye en la garantía de la sostenibilidad de las acciones en este orden.

- Se ha dotado al distrito de Cachí de una gama de servicios multidisciplinarios, en tres ejes interrelacionados: fortalecimiento de la percepción del riesgo, construcción de herramientas para la reducción del riesgo y fortalecimiento de la capacidad de gestión de la Red Local. Estas acciones ha coadyuvado en la gestión de algunas de las alternativas para la transformación de los escenarios de riesgo y en el funcionamiento de la Red Local para la reducción de riesgos en Cachí, en la cual la Universidad de Costa Rica figura como actor y fuente de insumos para apoyar el accionar de algunas de las iniciativas locales.

El siguiente diagrama ilustra las estrategias, interrelacionadas entre sí, que desde el año 2002 se han emprendido en el distrito de Cachí, en procura de la problematización del riesgo y de la generación de estrategias locales que prevengan los desastres en esa localidad, proceso en el cual ha sido significativa la participación de los actores sociales del distrito, como base y motor de las acciones propuestas: 


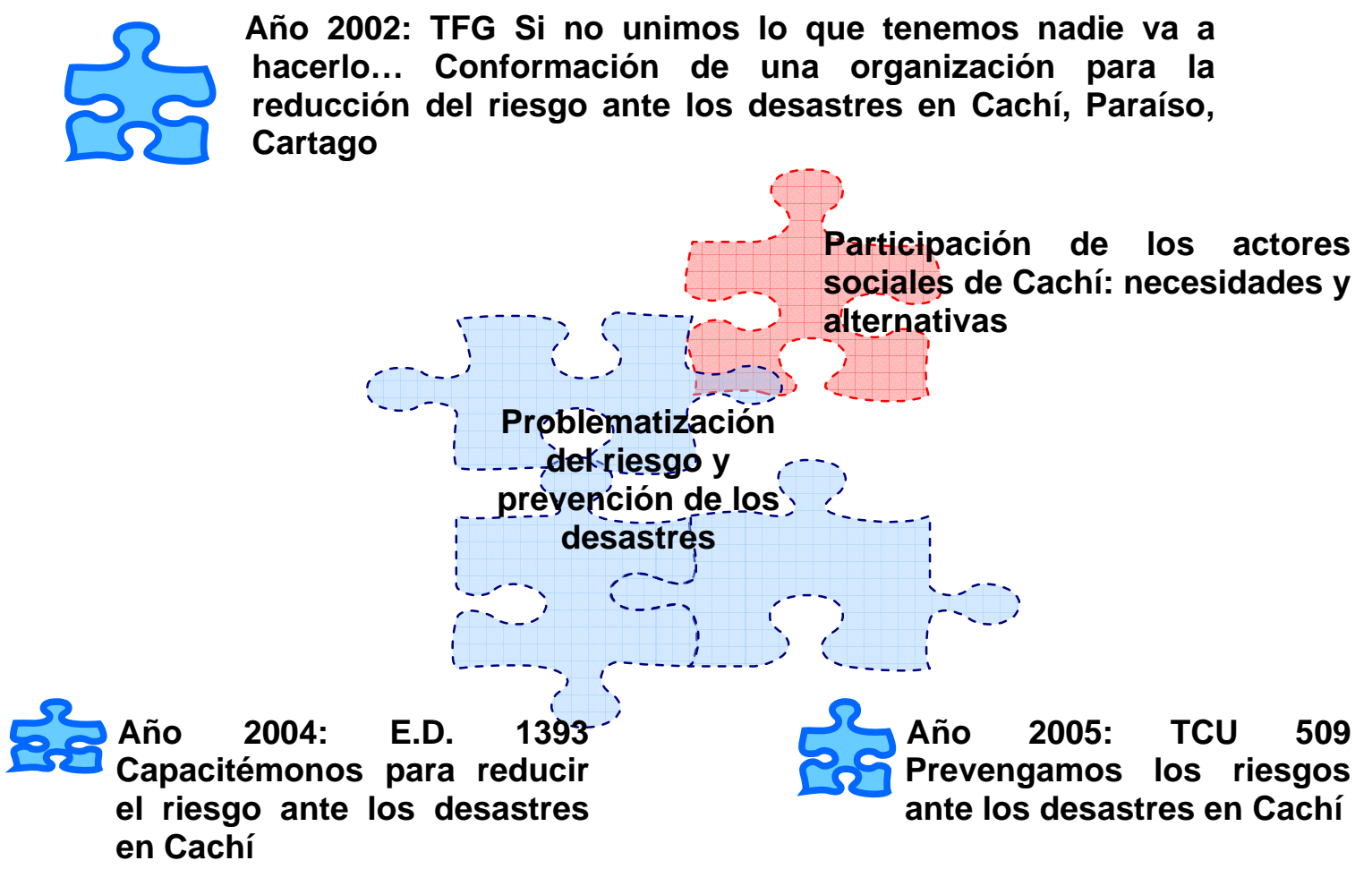

3. Articulación de la producción de conocimiento de los Trabajos Finales de Graduación (TFG) a las áreas críticas como puntos de partida para la investigación docencia y acción social.

Esta forma emerge como una articulación que permite realizar una mayor profundización del conocimiento que se deriva de los trabajos finales de graduación en relación con las áreas críticas.

En este sentido, la necesidad de la captación e intervención oportuna sobre las necesidades de la sociedad costarricense, lo mismo que sus problemas esenciales, se constituye en un imperativo para justificar la razón de ser de la formación académica de la Escuela de Trabajo Social. Las fórmulas para obtener este diagnóstico social han sido una constante en planificación estratégica y operativa de esta unidad académica.

La síntesis de "Áreas Críticas" que se explicitan como ejes para la acción de la docencia, la investigación y la acción social, se constituye en un esfuerzo investigativo, que se inició desde 1993. Los primeros estudios analíticos vislumbraron como prioritarios los problemas sociales, que posteriormente fueron complementados con discusiones y análisis sobre el Volumen 7, Número 1, Año 2007, ISSN 1409-4703 10 
conjunto de necesidades sociales relevantes para el Trabajo Social. En este sentido, los principales argumentos se refirieron a las posibilidades del Trabajo Social sobre el conocimiento e intervención en las necesidades, en los problemas, y en el fortalecimiento de las situaciones de ventaja social, y no solo circunscribir la acción académica a la atención de riesgo social.

Las áreas críticas se conciben como rutas de conocimiento y de intervención que constituyen puntos de partida para el quehacer académico de la formación académica.

Al respecto se definieron para el quinquenio 1995-2000 seis áreas denominadas:

- De la pobreza a la calidad de vida en igualdad de oportunidades

- Del deterioro de la salud a la salud integral

- Del deterioro del ambiente hacia el desarrollo sostenible

- De la crisis del Estado de Bienestar hacia la reforma del Estado

- De la violencia social a la democratización de la vida cotidiana y la convivencia social

- Del autoritarismo y la exclusión a la participación social y la ciudadanía plena

Por ello, se privilegió el estudio de las áreas:

- De la pobreza a la calidad de vida, en igualdad de oportunidades

- Del deterioro de la salud a la salud integral

- Del deterioro del ambiente hacia el desarrollo sostenible

- De la violencia social a la democratización de la vida cotidiana y la convivencia social

En un primer momento, esta investigación evidenció dos formas originarias que desarrolla la Escuela de Trabajo Social, para ofrecer un panorama que sirve de punto de partida para el estudio de los problemas sociales enunciados. La primera se refiere a la síntesis que se halla en el documento Áreas Críticas y otra emanada del análisis de los Trabajos Finales de Graduación.

Si bien es cierto este esfuerzo analítico es un primer eslabón en el planteamiento de la necesidad de sistematizar y reflexionar sobre los problemas señalados, también se constituye en un imperativo para reconocer, en los trabajos finales de graduación, la producción de conocimiento resultante, los aportes al conocimiento ya acumulado y en 
particular al documento de las áreas críticas. Estos son considerados la manifestación de un proceso académico que realizan los estudiantes para convertirse en acreedores del título. Es un tipo de tarea mucho más compleja que cualquier otra actividad realizada durante su carrera. Su función fundamental es la utilización de conocimientos y experiencias obtenidas. También se considera una instancia de producción de conocimiento nuevo, en lo que se refiere al conocimiento de problemas sociales (origen y evolución) y las formas efectivas de intervención en estos.

En relación con las áreas críticas, constituyen una fuente de temas para la elaboración de las propuestas de Trabajos Finales de Graduación, lo que permite trascender el conocimiento inicial descrito en estas. Sin embargo, este avance académico no es visible, ya que no existen los mecanismos para lograr la efectividad de la retroalimentación entre el conocimiento que se sintetiza en el documento de áreas críticas y la producción de los Trabajos Finales de Graduación.

La cuestión central que da sentido a este estudio está constituida por la necesidad de reconocer la producción de conocimiento emanada del quehacer académico de la Escuela de Trabajo Social. Sin embargo, solo una parte de esta producción es aprovechada. Se producen conocimientos en los cursos denominados teóricos en torno al estudio de la realidad nacional, el papel del Estado, la administración de los programas sociales y de las organizaciones comunales. Los cursos denominados talleres brindan la oportunidad de un conocimiento directo de la realidad. Se privilegian el conocimiento de problemas macro y micro sociales de Costa Rica, la intervención con grupos, organizaciones y comunidades; la intervención en programas institucionales y por último interesa el diagnóstico, la formulación y evaluación de los programas sociales (Campos, 2002). Para la realización de todo lo anterior, se retomaron como unidades de análisis los trabajos finales de graduación producidos en los períodos 1997-2000 y el documento de Áreas Críticas. Este último se adopta como un punto de partida y constituye una herramienta teórica para la toma de decisiones de la docencia, la investigación y la acción social.

Se efectuó un análisis de veintinueve trabajos finales de graduación, inscrito en la Vicerrectoría de Investigación con el título "La producción de Trabajos finales de Graduación y su contribución a las áreas críticas planteadas por la Escuela de Trabajo Social (19772000), N²15-A1-143" (Campos, Casas, Gutiérrez y Smith, 2003). El criterio para la 
selección de estos fue la denotación del contenido del título, en relación con el eje que contempla el área crítica. Es importante subrayar que un total de 8 trabajos expresan en su título temas diferentes a los explícitos en el área crítica. Al respecto, la siguiente tabla destaca el número de trabajos finales de graduación que se revisaron en relación con el área:

\begin{tabular}{lc}
\hline De la pobreza a la calidad de vida, en igualdad de oportunidades & 9 \\
Del deterioro de la salud a la salud integral & 4 \\
$\begin{array}{l}\text { Del deterioro del ambiente hacia el desarrollo sostenible } \\
\text { De la violencia social a la democratización de la vida cotidiana y la }\end{array}$ & 5 \\
Convivencia & \\
Del autoritarismo y la exclusión a la participación social y la ciudadanía & 6 \\
plena & 29 \\
Total & \\
\hline
\end{tabular}

Los principales resultados giraron en torno a la profundización de distintas aristas relacionadas con las áreas críticas:

En relación con el área de la pobreza a la calidad de vida, en igualdad de oportunidades, los trabajos finales muestran la necesidad del estudio y análisis de la pobreza como un problema estructural que genera otros problemas relacionados con la exclusión .Lo anterior permite plantear la necesidad de profundizar sobre los aspectos conceptuales y empíricos de la pobreza en Costa Rica, la función del Estado costarricense y los programas relacionados con la pobreza.

Otro tema que adquiere relevancia es el que se refiere al estudio de la familia en Costa Rica. Con el propósito de profundizar en el manejo de la teoría que existe sobre el estudio de las familias, se privilegió este conocimiento. El anterior fue uno de los aspectos al que el equipo de investigadores dio un peso importante, debido las dificultades encontradas por su omisión. Esta situación originó un fuerte argumento para recomendar la urgente necesidad de efectuar estudios relacionados con las teorías sobre familia que predominan en la formación académica. Al respecto se concretan algunos aspectos a tomar en cuenta en este estudio: una descripción de datos empíricos sobre las características de la familia costarricense y los principales cambios que se expresan en la actualidad, el perfil actual de 
las familias y las configuraciones familiares como una forma de conocer las manifestaciones de los cambios de las familias actuales.

En relación con el área llamada "Del deterioro de la salud a la salud integral", se abordó la importancia del modelo de salud basado en la prevención y atención primaria. Se reconoce que los nuevos paradigmas en salud constituyen posibilidades de acción para el Trabajo Social. Esto por cuanto la influencia predominante del modelo médico permea la intervención del Trabajo Social. Una recomendación de fuerza que emerge de este apartado se relaciona con que el Trabajo Social ofrece las herramientas teóricas y metodológicas para la intervención en el fortalecimiento y desarrollo de la salud. Esto fundamenta las posibilidades de acción del Trabajo Social en la prevención y participación social en salud.

En el área llamada "Del deterioro del ambiente hacia el desarrollo sostenible", se trabajó el concepto de ambiente y su importancia para el Trabajo Social. No obstante, los aportes de trabajos finales de graduación en esta área han sido muy escasos. Por lo se plantea como recomendación el estudio sobre este tema.

En relación con el área llamada "De la violencia social a la democratización de la vida cotidiana y la convivencia social", en este estudio se condensan tres conjuntos de conocimientos. El primero explicita los aspectos que contempla el área crítica sometida al examen; una descripción de los conocimientos que se aportan al área y por último se condensa el conocimiento desde la perspectiva de la investigadora.

Otro tema de relevancia que se estudia es el de la participación social, como vital para la intervención del trabajador social. El involucramiento estatal en la gestación de políticas sociales crea una serie de premisas sobre las que descansa la participación social. En este apartado se profundiza en el concepto de desarrollo participativo, el cual implica que las personas que compartan problemas mutuos, deben tener la confianza de plantear las responsabilidades para expresar y reclamar sus demandas y trabajar en forma conjunta en la búsqueda de soluciones.

Una de las conclusiones importantes en esta experiencia articuladora se relaciona con la estrategia que se ha privilegiado en la Escuela de Trabajo Social a partir del planteamiento de las áreas críticas como un valioso punto de partida. En este sentido, se sitúan en la 
agenda de discusión temas de interés social y que expresan demandas de colectivos sociales a los que el Trabajo Social debe responder como parte de su legitimidad. En el caso de estudios particulares, como los trabajos finales de graduación, permiten generar conocimientos singulares que ilustran sobre la especificidad de los problemas sociales y la diversidad de sus contextos

\section{Lecciones aprendidas}

1. Las experiencias desarrolladas expresan con fidelidad la importancia y la necesidad de la articulación de procesos de la investigación, la docencia, y la acción social. También es fundamental reconocer que la participación activa de los actores en la reflexión y la transformación de situaciones sociales que generan asimetrías sociales, se constituye en una práctica social que va permitir la transformación de la realidad a partir de la acción de los sujetos en esta relación.

2. La necesaria revisión en forma permanente de los postulados epistemológicos y metodológicos en las formas de investigación e intervención del Trabajo Social. El contacto cercano que tiene nuestra profesión con la realidad, tal como la viven las personas, nos permite captar ópticas diferentes de los problemas sociales. Cuanto más a fondo sea el conocimiento del problema, mayor es la posibilidad de abordarlo correctamente. De esta manera, la producción de conocimiento puede incidir en los lineamientos de política social, de tal forma que sean una respuesta a prioridades sociales. Al respecto, la información concreta que poseen las personas puede servir de índice de realidad y como guía para el diseño de políticas que incluyan como principio fundamental el respeto de los derechos de las personas. La Escuela de Trabajo Social, como unidad formadora, tiene la responsabilidad social de vigilar la calidad de la producción de conocimiento que emerge de la academia. Tal situación debe resultar en un ejercicio profesional con una alta motivación para el despliegue de investigaciones que redunden en intervenciones de alto rigor científico y, consecuentemente, de impacto para las poblaciones en riesgo social, a partir de la incidencia de políticas que desemboquen en una mejor calidad de vida. 
3. Lo señalado nos permite entonces explicitar una inicial aproximación teórica y metodológica de lo que se denomina núcleo, como integrador de la investigación, la docencia y la acción social. El núcleo puede definirse como:

- El desarrollo de la capacidad de reflexión sobre determinados aspectos de la realidad social, pero tal reflexión al mismo tiempo depende de las condiciones sociales en las que se desarrolla el núcleo, de los propósitos y las características que este adopta.

- Supone un proceso de interacciones, en al menos tres dimensiones de relaciones sociales. Interesa destacar la articulación de la docencia, la investigación, la acción social; la de estudiantes, docentes, profesionales, grupos y personas con las que se trabaja, y la teoría y práctica.

- El núcleo se puede conceptualizar como la unidad estructural en la que descansa la articulación de las actividades académicas en la tripleta citada. Tales actividades constituyen una totalidad que se manifiesta en el proceso de producción de conocimientos. Sin embargo, para efectos de delimitación se entenderá lo siguiente:

Investigación: es el momento productivo del conocimiento que supone una captación progresiva y sistemática de las expresiones de la realidad social particular en la cual se desenvuelve la praxis social.

Docencia: considerada como un momento de alimentación e inversión de los insumos culturales que favorecen un ordenamiento conceptual preliminar de la realidad social, cuyos rasgos más sobresalientes convergen en los aspectos formativos del núcleo. Tales aspectos están orientados por ciertas directrices ${ }^{4}$ que se apoyan permanentemente en la investigación y la acción social. Esta es realizada por la comunidad de profesores estudiantes y profesionales.

Acción social: es la proyección externa de las actividades que realizan estudiantes y profesores a diferentes niveles de la realidad social en la que se han insertado. Las modalidades de esta acción contemplan actividades con profesionales, grupos y personas con los que se participa en los procesos académicos.

\footnotetext{
${ }^{4}$ Tales directrices constituyen el plan de estudio o estructura curricular en donde se esbozan los desafíos contextuales y el conjunto de aspectos contradictorios que se gestan entre los planteamientos formales y la realidad misma. 
La integración de tales actividades supone lo siguiente:

- la superación de la compartimentalización curricular en asignaturas teóricas y prácticas o en la aplicación práctica de los cursos teóricos.

- la superación de una orientación pedagógica centrada en la transmisión de conocimientos de la realidad en forma yuxtapuesta a una que se centra en la captación de contradicciones. Tal captación, sin embargo, más que una enunciación taxonómica de tipos de contradicciones, remite a un proceso que, a partir de la práctica misma, confluye en la delimitación de los elementos contrapuestos de diversos grados de profundidad, cuya síntesis genera momentos efectivos de conocimiento.

- la sustitución de relaciones verticales entre estudiantes, profesionales profesores y personas atendidas en los servicios institucionales, en las comunidades $y$ en sus familias, por relaciones horizontales en la transformación de situaciones que favorezcan el cumplimiento de servicios estatales a los que tienen derecho.

- la superación de una acción espontánea limitada a una acción científica y transformadora de las situaciones que generan desigualdades sociales y la inaccesibilidad a los derechos sociales.

- la superación de intereses particulares focalizados por intereses colectivos y universales consonantes con los fines de la misión y visión de la Escuela de Trabajo Social y la Universidad de Costa Rica.

4. En consonancia con lo anterior, es importante reconocer que la esencia del núcleo se genera a partir del desarrollo progresivo de la articulación de procesos académicos como una posibilidad considerada vital para la sobrevivencia de la organización académica (Campos, 1991). En este sentido el desarrollo de articulaciones del proceso formativo profesional no se limita a la transmisión rutinaria de verdades absolutas o al cumplimiento formalmente escolar de actividades aisladas del contexto social, sino que, por el contrario, crece dentro de la multiplicidad de relaciones sociales. Por ello, la investigación se constituye en una de las mediaciones para aprehender el movimiento de la realidad y sus contradicciones y la acción social es la resultante y resultado del proceso anterior en la búsqueda específica del proceso de transformación social, con la participación de docentes, estudiantes, profesionales y las personas sujetos de derechos sociales. 
En consecuencia, el proceso de la docencia sintetiza la investigación y la acción social Sin embargo, este proceso no se explica solo por las características aisladas de los componentes humanos y estructurales, sino que debe remitirse al conjunto de condiciones en las cuales se realizan determinadas relaciones sociales de dirección recíproca. Un conjunto de estas condiciones remite al desarrollo histórico; fueron adquiridas por formas de expresión concretas a través del Estado y de sus instituciones, en el sentido de que tales condiciones determinan en forma predominante los propósitos de la formación académica. Otro conjunto de condiciones de mayor particularidad son las ubicadas en la Universidad, las cuales resumen proyectos universitarios innovadores que sintetizan movimientos de crítica y de superación de las condiciones actuales, que posibilitan proyectos de carácter integral, como las experiencias expuestas.

\section{Algunas consideraciones finales}

Este artículo presentó en su desarrollo la desarticulación de las actividades académicas, como uno de los problemas fundamentales para el planteamiento de los núcleos. También fue relevante reconocer en los esfuerzos académicos las posibles condiciones, los caminos para el desarrollo actual de los NIDIAS. Este recuento permite realizar unas sugestiones en relación con estas formas originarias denominadas NIDIAS:

La práctica académica en la formación del Trabajo Social comprende el escenario vital para el despliegue de rutas de procesos de investigación y acción social. En estas, estudiantes, profesores, pobladores de las comunidades y funcionarios de las instituciones asumen roles protagónicos en la toma de conciencia y transformación de las situaciones que afectan su calidad de vida.

La naturaleza del Trabajo Social lleva implícita la necesidad de que el proceso de conocimiento se construya en la actividad práctica, y no en la contemplación e intuición. El trabajo social, como disciplina y profesión, manifiesta en su esencia la cualidad de condensar en cada uno de sus procesos académicos la investigación y la acción social.

Es fundamental la reflexión, la investigación de las prácticas que se realizan, de tal manera que no desemboquen en utilitarismo, cuyos intereses solamente permitan un conocimiento fenoménico, una orientación en el mundo pero no una comprensión de las cosas. Las prácticas fragmentadas expresan un mundo de fenómenos externos que se desarrollan en la 
superficie de la realidad. Tal concepción implica que las modificaciones planteadas afectan la apariencia y no la esencia; por ello la articulación debe responder a una práctica transformadora y transformada por los sujetos mismos. En este sentido, la práctica del Trabajo social debe partir del reconocimiento de nuevas cuestiones sociales, el rescate de las experiencias de la población subalterna, que plantea acciones cuestionadoras y soluciones a problemas sociales cada vez más agravados por la concentración de riquezas, discrepancias de oportunidades culturales y las distorsiones en las manifestaciones ideológicas y políticas.

En consecuencia, es importante subrayar la función determinante que tiene la articulación como soporte estructural en el proceso académico. No obstante, esta tiene formas distintas de expresión $y$, por ende, de aprehenderse. Con el propósito de asumir aquellas que trasciendan los aspectos fenoménicos aparenciales, se deben contemplar algunas categorías iniciales para la comprensión de la articulación. Al respecto, es relevante preguntarse ¿cuál es la concepción de realidad que subyace detrás de cada proceso de articulación? Pueden encontrarse distintas respuestas que coexisten al margen o en forma dominante.

En síntesis, se señalan tres concepciones de realidad que coexisten, se interpenetran y generan consecuencias importantes en la praxis social de los sujetos participantes en la práctica académica y profesional. Una de ellas expresa una concepción de realidad como totalidad fragmentada; otra otorga prioridad y predominio del todo sobre las partes, y otra como un todo estructurado que se desarrolla y crea. En la primera pueden identificarse los aportes desde Descartes a Wittgenstein; la otra concepción denominada organicista y organicista -dinámica que formaliza el todo y afirma el predominio y la prioridad del todo sobre las partes, desde Shelling a Spann y la concepción dialéctica desde Heráclito, Hegel y Marx, que concibe la realidad como un todo estructurado que se desarrolla y crea (Kosik, 1976, p. 64).

A partir de la respuesta a lo anterior es fundamental derivar la comprensión de la actividad práctica que predomina en el Trabajo Social como punto de partida para desarrollar una praxis transformadora de las situaciones de desigualdad e inequidad social. Consecuentemente, la articulación de los procesos de investigación, docencia y acción social deben partir en forma imperativa de una concepción de realidad que se va creando y 
desarrollando, de tal manera que la complejidad y las transformaciones se constituyan en ejes de la investigación, la docencia y la acción social.

\section{Referencias}

Campos, Ivette; Casas, Gerardo; Gutiérrez, Hernán; Smith; Marjorie. (2003). La producción de los trabajos finales de graduación y su contribución a las áreas críticas planteadas por la Escuela de Trabajo Social (1997-2000) (Proyecto de investigación No 215-A1-143). San José: Escuela de Trabajo Social. Universidad de Costa Rica.

Campos, Ivette; Norma, Méndez. (2003). La integración de la investigación, docencia y acción social en torno a la democratización de la vida cotidiana, como alternativa de acción para la atención de la violencia social (Proyecto de Investigación $N^{\circ} 215-$ Al-089). San José: Escuela de Trabajo Social. Universidad de Costa Rica.

Campos, Ivette. (2002). La práctica académica como eje para la integración de la investigación y la acción social. Revista Reflexiones, 81(1). Facultad de Ciencias Sociales. Universidad de Costa Rica.

Campos, Ivette. (2002). La función de la investigación en el proceso académico de la Escuela de Trabajo Social. Revista Análisis, III(1). Escuela graduada de Trabajo Social Beatriz Lassalle. Facultad de Ciencias Sociales. Universidad de Puerto Rico.

Campos, Ivette. (1991). El concepto de integración y su puesta en práctica en la formación académica de la Escuela de Trabajo Social de la Universidad de Costa Rica. Revista Costarricense de Trabajo Social. (1). Órgano Oficial del Colegio de Trabajadores Sociales, Costa Rica.

Campos, Ivette; Chinchilla, Marisol y Molina, Emilia. (1995). Áreas Críticas. Documento de trabajo Escuela de Trabajo Social. Universidad de Costa Rica. Costa Rica.

Cerdas, Laura. (2005). Prevengamos los riesgos ante los desastres en Cachí, Paraíso Cartago [Informe final Proyecto de Trabajo Comunal, TC -509]. San José, Costa Rica: Escuela de Trabajo Social, Universidad de Costa Rica.

Escuela de Trabajo Social. (2001). Informes de Labores. 1999-2000. San José: Asamblea de Escuela. Universidad de Costa Rica.

Escuela de Trabajo Social. (2004). Plan de estudios 2004-2010. San José: Universidad de Costa Rica.

Kosik, Karel. (1976). Dialéctica de lo concreto. México: Editorial Grijalbo.

Matus, Teresa. (1995). Desafíos del Trabajo Social en los 90. Perspectivas metodológicas en Trabajo Social. Chile: ALAETS-CELATS.

Universidad de Costa Rica. (1990). Estatuto Orgánico. San José, Costa Rica: Oficina de Publicaciones. 